\title{
A Portable Fixed-Target Sample Delivery System for in-situ Serial Crystallography
}

\author{
A Finke ${ }^{1}$, G Illava ${ }^{2}$, B Apker ${ }^{3}$, R Jayne ${ }^{4}$, D Closs ${ }^{4}$, Q Huang ${ }^{5}$, I Kriksunov ${ }^{5}$, S Milano ${ }^{5}$, D Schuller ${ }^{6}$, D \\ Szebenyi ${ }^{6}, \mathbf{R}$ Cerion $^{5}, \mathrm{R}$ Thorne $^{7}$ \\ ${ }^{1}$ Cornell University, Groton, NY, ${ }^{2}$ Cornell, Ithaca, NY, ${ }^{3}$ MiTeGen, ${ }^{4}$ MiTeGen, Inc., Ithaca, NY, \\ ${ }^{5}$ Cornell University, Ithaca, NY, ${ }^{6}$ MacCHESS, Cornell Univ., ${ }^{7}$ Physics Dept, Cornell Univ. \\ af554@cornell.edu
}

Protein crystallography enables atomic-level structure determination from crystalline specimens. Due to the rapid onset of radiation damage at room temperature, the vast majority of protein crystal structures are done at cryogenic temperatures. While cryocooling alleviates much of the practical issues of radiation damage, it also restricts the energy landscapes in which proteins normally exist, which can lead to inaccurate pictures on the room temperature dynamics of conformation, allostery, and substrate binding. We have developed a scalable, portable fixed-target delivery system for serial crystallography that enables rapid data collection with minimized background. Ultrathin Kapton platforms are photopatterned to create wells that can localize crystals into a regular grid, and remaining mother liquor can be removed by vacuum or wicking with a paper towel to minimize background. For sensitive or difficult crystals, these platforms are also highly amenable to in-situ crystallization methods. The platform design allows crystal growth to be localized around well spaces, which simplifies data collection by rastering. We have demonstrated the utility of our new design on a number of novel drug-binding proteins.

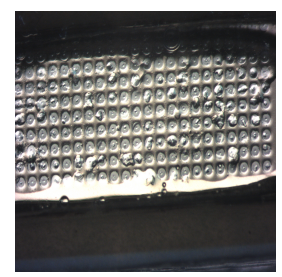

Figure 1

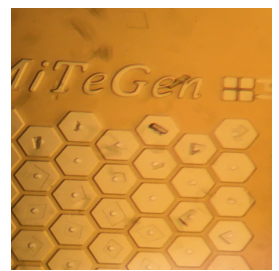

Figure 2

Acta Cryst. (2020). A76, a102 